XIV Simpósio Nacional de Sistemas Prediais

Gestão, Eficiência e Sustentabilidade

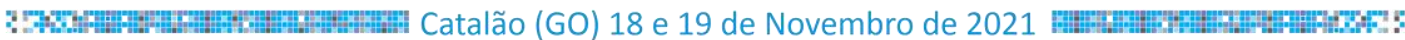

\title{
GESTÃO E CONCEPÇÃO DOS PROJETOS HIDROSSANITÁRIOS INTEGRADOS COM OS DEMAIS PROJETOS ELABORADOS NA CIDADE DE GOIÂNIA-GO
}

\section{Management and design of plumbing systems integrated with other projects developed in the city of Goiânia-GO}

\author{
AMARAL, Letícia Leal'ㄹ PITALUGA, Douglas Pereira da Silva²
}

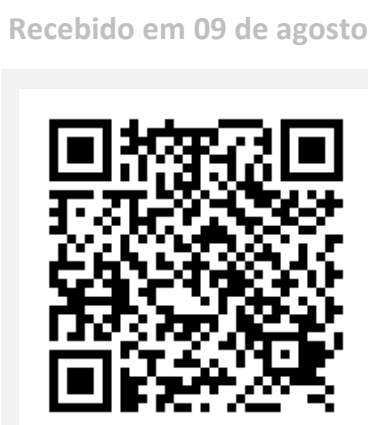

Palavras-chave:

Projetos de Sistemas Hidrossanitários,

Coordenação de projetos,

Fases do projeto, Projeto na engenharia civil.

\section{Keywords:}

Plumbing design project coordination;

Project phases;

Design in

civil engineering.
RESUMO: Diante as dificuldades e os problemas relacionados à gestão de projetos no âmbito da construção civil, é almejado com este trabalho entender como as empresas localizadas na cidade de Goiânia se organizam durante o processo de elaboração dos projetos hidrossanitários em conjunto com os demais projetos existentes das edificações, a fim de identificar a real contribuição de aspectos facilitadores como a atuação dos coordenadores de projetos e daqueles que possam comprometer com os resultados. A metodologia utilizada para este estudo deu-se a partir de entrevistas realizadas com empresas projetistas da cidade, visando compreender a gerência de elaboração dos projetos hidrossanitários para os diversos edifícios em obra, enfatizando o trabalho simultâneo dos projetistas de diferentes áreas. A partir da análise dos resultados obtidos, pôde-se identificar se existe um padrão quanto a elaboração dos projetos; identificar as fases pelas quais os projetos hidrossanitários passam durante sua concepção; entender como funciona a comunicação entre os projetistas de diferentes áreas; e identificar o reflexo da atuação dos coordenadores de projetos nos resultados das empresas.

\begin{abstract}
In view of the difficulties and problems related to project management in the scope of civil construction, it is aimed with this work to understand how companies located in the city of Goiânia are organized during the process of elaboration of plumbing systems projects in conjunction with the other existing projects of buildings, in order to identify the real contribution of facilitating aspects such as the role of project coordinators and those who may compromise with results. The methodology used for this study was based on interviews with design companies in the city, aiming to understand the management of the elaboration of plumbing systems projects for the different buildings under construction, emphasizing the simultaneous work of designers from different areas. From the analysis of the results obtained, it was possible to identify if there is a standard regarding the elaboration of the projects; identify the phases that the plumbing systems projects go through during their conception; understand how communication works between designers in different areas; and to identify the reflex of the performance of the project coordinators in the results of the companies.
\end{abstract}

CONTATO DOS AUTORES:

${ }^{1}$ AMARAL, Letícia Leal: Instituto Federal de Goiás, leticialealamaral@gmail.com.

2 PITALUGA, Douglas Pereira da Silva: Instituto Federal de Goiás, douglas.pitaluga@ifg.edu.br. 


\section{INTRODUÇÃO}

Face a dinâmica das mudanças tecnológicas e o movimento do setor da construção civil para melhoria da qualidade dos processos produtivos, tem-se observado nos últimos 20 anos que as empresas do setor têm mudado suas concepções diante a importância do processo de produção de projetos (WOOD Jr., 1993). Estes devem cada vez mais serem inovadores e adequados às necessidades atuais, atendendo às expectativas dos incorporadores e consumidores, não só em qualidade, mas também em sustentabilidade, eficiência e produtividade.

Em vista dos altos padrões de qualidade exigidos no processo de produção dos projetos, somado ao aumento de complexidade e ao pouco tempo de elaboração disponibilizado às empresas, o mercado da construção de edifícios vem contando com a atuação de diferentes profissionais e empresas distintas para trabalharem em conjunto, mesmo estando em locais diferentes (NÓBREGA e MELHADO, 2013). Com isso, a figura do gestor e coordenador de projetos se tornou cada vez mais importante e necessária, atuando como uma "ponte" para troca de informações entre os profissionais.

De acordo com Helene (2003), os erros de projetos são responsáveis por cerca de $40 \%$ dos problemas relatados no período de assistência pós-obra. Em pesquisa realizada por Brandão (2007), tem-se que o principal motivo de chamado para reparo feito pelos usuários durante o período de assistência, são os problemas relacionados aos sistemas prediais hidrossanitários, responsáveis por cerca de $9 \%$ das ocorrências e com problemas distribuídos entre: retorno de gases, vazamentos, obstruções nas tubulações, retorno de espuma, vazamento em ralos.

Quando se verifica os diversos estudos sobre os processos de elaboração dos variados projetos na indústria da construção civil, têm-se que as patologias na construção, em sua maioria, são consequências do processo de elaboração dos projetos (FIESS et al, 2004). De acordo com pesquisas realizadas pelo autor, a fase de projeto delimita em torno de $35 \%$ das principais causas dos problemas patológicos.

Portanto, a relevância do tema de gestão do processo de elaboração dos projetos, do ponto de vista mercadológico, é bastante relevante, pois, é na fase de projeto que se evita grande parte dos problemas gerados durante e após a execução da obra, logo, este trabalho se justifica.

\section{OBJETIVOS}

O objetivo deste trabalho foi identificar o reflexo das ações dos coordenadores de projetos no número de problemas patológicos ocorridos no período pós-obra. Caracterizando, assim, como funciona a gestão de projetos e atuação dos seus coordenadores durante a elaboração dos projetos hidrossanitários na cidade de Goiânia. Bem como, identificando a estrutura organizacional das fases de produção que um projeto hidrossanitário passa até ser entregue ao cliente e, por fim, caracterizando a forma de comunicação e troca de informação entre os profissionais de projetos de diferentes áreas.

\section{FUNDAMENTAÇÃO}

Nos empreendimentos de engenharia, a motivação pela implantação de um sistema da qualidade deve estar predominantemente vinculada à redução de custos finais dos produtos, à diminuição das perdas no processo de produção e aos custos de retrabalho e correções pós- 
entrega (MELHADO, 1994). Logo, pensando em qualidade de produto na construção civil, darse-á importância não só à fase de execução da obra, como também à fase de planejamento e projeto.

Levando em conta a importância do sistema hidrossanitário sob a ótica de desempenho, Borges (2008) mostra que esses sistemas, além de exercer sua função específica de abastecer de maneira adequada os usuários com água quente e fria, deve também ter a capacidade, entre outras funções, de absorver as deformações e esforços gerados pelos outros sistemas inter-relacionados, notadamente a estrutura do edifício. O desempenho de um sistema, portanto, afeta outros sistemas e vice-versa, e o desempenho global deve ser encarado como um todo integrado.

Essa colaboração de diferentes profissionais, como ainda afirmaram Nóbrega e Melhado (2013) é fundamental para o sucesso do projeto final, porém traz consigo diversas dificuldades relativas à coordenação dos projetos e dos projetistas. Nesse contexto, o coordenador de projetos surge como agente fomentador não apenas da interação e cooperação entre todos os agentes envolvidos no processo de projeto, mas também do bom resultado do processo e das soluções de projeto adotadas. (NÓBREGA JR. e MELHADO, 2013).

Já no assunto de compatibilização de projetos, Silva e Novaes (2008) entendem esta como uma atividade necessária para que as diversas soluções dimensionais, tecnológicas e estéticas sejam compatíveis entre si e no conjunto dos projetos. Após a avaliação das soluções propostas nos diversos projetos, ela se processa por meios manuais ou digitais. É realizada a superposição e análise de desenhos impressos ou digitais em pranchas bidimensionais ou em 3D. A compatibilização de projetos se inicia na fase de estudos preliminares, prossegue na fase de anteprojetos e termina com a compatibilidade parcial e final na fase dos projetos executivos.

\section{MÉTODO}

A pesquisa aqui apresentada tem finalidade básica-estratégica e objetivo exploratório. Como método de pesquisa deste trabalho, foram feitas entrevistas via vídeo conferência (devido a pandemia do COVID-19) com o maior número de empresas de projetos disponíveis localizadas na cidade de Goiânia, com perguntas baseadas na referência bibliográfica aqui apresentada.

\section{RESULTADOS E DISCUSSÃO}

A partir da análise das respostas fornecidas pelos entrevistados, foi elaborado uma Tabela 1 indicativa que contém os tópicos das conversas que mais interessam neste trabalho. Indicado com " $X$ " as medidas ainda não adotadas pelas empresas. Não há indicativos de certo ou errado. 
Tabela 1 - Medidas ainda não adotadas pelas empresas (indicadas com " $X$ ")

\begin{tabular}{|c|c|c|c|c|}
\hline \multirow{2}{*}{ DESCRIÇÃO DE CARACTERÍSTICAS } & \multicolumn{4}{|c|}{ EMPRESA } \\
\hline & 01 & 02 & 03 & 04 \\
\hline Coordenador de projetos com função exclusiva; & $\mathrm{X}$ & $\mathrm{X}$ & $\mathrm{x}$ & $\mathrm{x}$ \\
\hline Informações de projetos centralizadas & $\mathrm{X}$ & SIM & $\mathrm{x}$ & SIM \\
\hline Plataformas de comunicação entre projetistas; & $\mathrm{x}$ & SIM & $\mathrm{x}$ & $\mathrm{x}$ \\
\hline Fases para elaboração de projetos hidrossanitários bem definidos; & SIM & $+/-$ & $\mathrm{x}$ & $\mathrm{x}$ \\
\hline Projetistas hidrossanitários contratados na empresa & SIM & SIM & $\mathrm{x}$ & $\mathrm{x}$ \\
\hline Soluções dos projetos tomadas coletivamente & SIM & SIM & $\mathrm{x}$ & $\mathrm{x}$ \\
\hline Compatibilização de projetos em modelagem 3D & SIM & SIM & SIM & $\mathrm{x}$ \\
\hline Acompanhamento de obras por parte da equipe projetista & $\mathrm{x}$ & $\mathrm{x}$ & $\mathrm{x}$ & $\mathrm{x}$ \\
\hline Assistência técnica durante a obra & SIM & SIM & SIM & SIM \\
\hline
\end{tabular}

Fonte: Próprio do autor, 2020.

Figura 1 - Elaboração dos projetos hidrossanitários das empresas consultadas

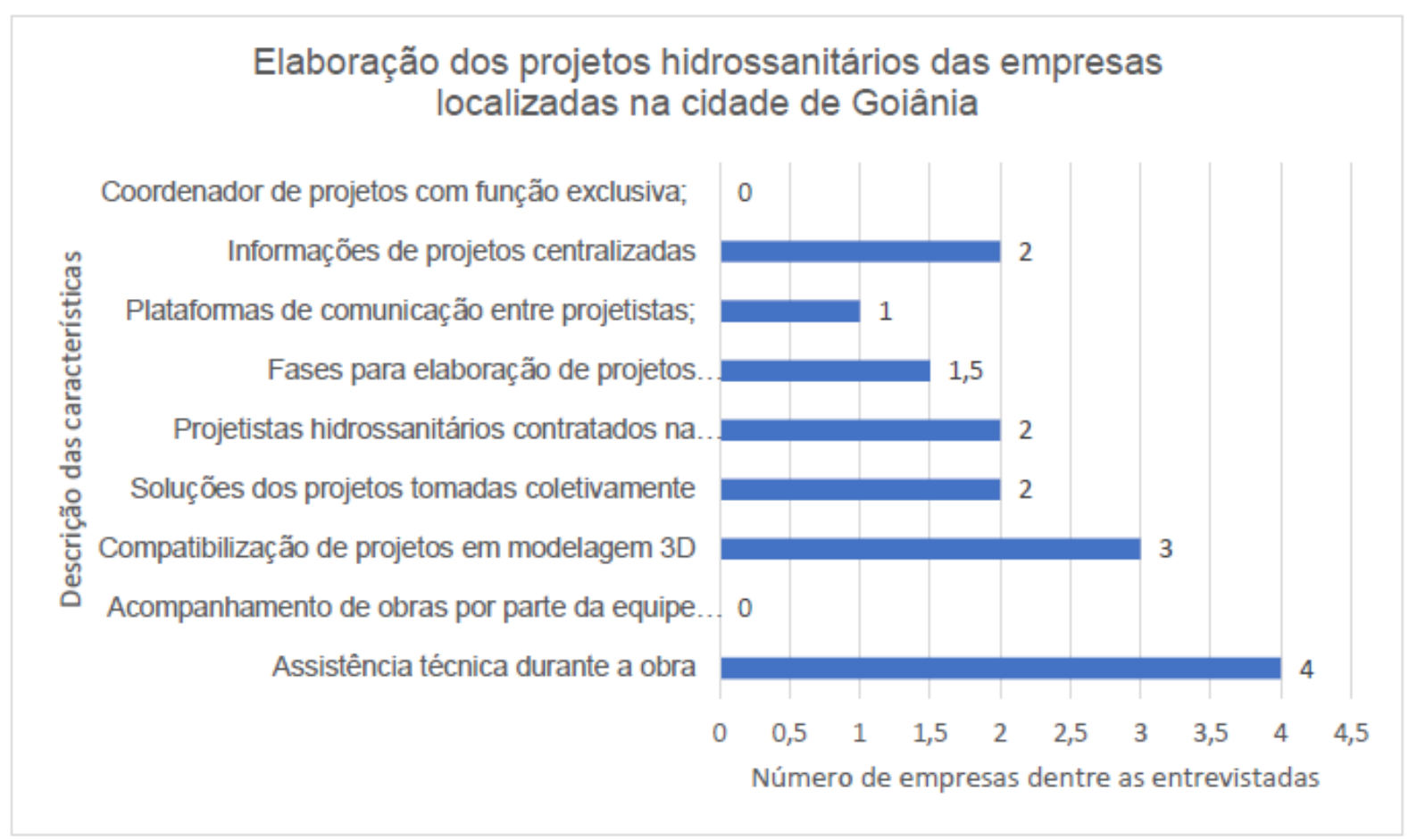

Fonte: Próprio do autor, 2020.

Verifica-se que nenhuma das empresas entrevistadas conta com a presença de um profissional coordenador de projetos de função exclusiva e especializado na área, porém todas as empresas possuem um profissional que desempenha, dentre várias funções, a de coordenador.

Apesar de todas as empresas possuírem um coordenador de projetos, apenas as empresas 2 e 4 optam por centralizar todo o contato com o cliente e recebimento de informações de projetos por esse único profissional. Observa-se ainda que somente a empresa 2 utiliza de 
uma plataforma de apoio na comunicação entre os projetistas, a fim de manter toda a equipe atualizada sobre as alterações que ocorrem comumente.

Quanto a elaboração dos projetos hidrossanitários em conjunto com os demais projetos da edificação, tem-se que somente a empresa 1 possui as fases de concepção da área bem definidas, enquanto que a empresa 2 tem-se essas fases parcialmente definidas, visto que possui algumas etapas pré-definidas, porém essas são um pouco confusas, podem mudar de acordo com o cliente e o contrato, e se misturam entre si.

As empresas 1 e 3 não possuem engenheiros com contratos fixos para elaborarem os projetos hidrossanitários, tais são terceirizados.

As empresas 1, 2 e 3 utilizam da tecnologia de modelagens 3D para compatibilização das diferentes áreas. Todas afirmam que essa compatibilização não deve ser feita somente no final do processo, mas sim durante toda a concepção.

Por fim, nenhuma das empresas entendem que seja necessário o acompanhamento em obras por parte da equipe de projetos, mas todas defendem que a empresa deve estar disponível para fornecer qualquer assistência que o cliente venha solicitar durante e após a obra, ou para tirar quaisquer dúvidas que podem surgir na leitura dos projetos durante a execução.

\section{CONSIDERAÇÕES FINAIS}

A partir da interpretação das respostas obtidas pelos entrevistados, é entendido que as empresas localizadas na cidade de Goiânia não seguem um certo padrão quanto aos desenvolvimentos dos projetos, principalmente quando verificado os projetos complementares, em específico o hidrossanitário.

A maneira como as empresas entrevistadas elaboram seus projetos hidrossanitários e determinam suas obrigações com os clientes, depende muito do tipo de cliente que está as contratando. Em casos onde se tem clientes privados, a elaboração dos projetos e os produtos apresentados aos clientes ao final de cada etapa, depende muito do que foi combinado em contrato, ou seja, do quanto o cliente está disposto a pagar.

De forma geral, os projetos hidrossanitários passam por fases de elaboração semelhantes às fases dos projetos de arquitetura, porém, nestes primeiros as fases são menos definidas e se misturaram mais entre si. A partir das entrevistas, conclui-se que os projetos hidrossanitários passam basicamente por 3 (três) fases comuns a todas as empresas, sendo essas: reunião de partida ou descoberta (1); estudos preliminares/anteprojeto (2) e projetos executivos (3).

A atuação dos coordenadores durante essa concepção dos projetos na cidade de Goiânia é bem sutil. As empresas afirmam ter um profissional responsável pela função de coordenador, entretanto este não está destinado a realizar somente as tarefas destinadas ao mesmo, e percebe-se que tal cargo não se destaca como a função principal dos colaboradores. A maioria dos coordenadores de projetos são os próprios sócios das empresas e, também, atuam como projetistas. Logo, entende-se que as empresas ainda não perceberam a necessidade desses profissionais na equipe e que ainda não se faz uma tradição de mercado na cidade.

Quanto a comunicação entre os projetistas, é constatado que as empresas encontram dificuldades na implementação de plataformas de apoio. A maioria das empresas ainda têm como principal troca de informações as mensagens informais, que não mantém um histórico organizado e não lembram os projetistas de todas as alterações de projetos adotadas a cada 
revisão. Logo, percebe-se que a dificuldade das empresas em implementarem essas novas tecnologias, estão diretamente relacionadas à falta do profissional responsável pela coordenação de projetos, este que deveria se dedicar exclusivamente a manter a equipe em perfeita sintonia.

A compatibilização dos projetos hidrossanitários com os demais projetos da edificação ocorrem em maioria das vezes durante todo o processo de elaboração dos mesmos. A maior parte das empresas entrevistadas já trabalham com projetos em modelagens 3D. Consta-se que é muito importante que a compatibilização dos projetos ocorra desde os estudos preliminares das soluções adotadas nos projetos hidrossanitários, ou seja, é preciso estar com os projetos arquitetônicos em mãos para planejar e começar a desenvolver os demais projetos. Percebe-se que é um senso comum entre as empresas projetistas que os projetos de arquitetura devam se manter inalterados.

Por fim, pode-se perceber com base nas respostas das empresas entrevistadas, que falta uma valorização da etapa de elaboração dos projetos. Essa etapa é comumente definida de acordo com cada contrato e muitas vezes os clientes não pagam por todos os projetos que a empresa tem a oferecer, sendo assim retratado o problema cultural do imediatismo brasileiro, descrito por vários autores e citados na revisão bibliográfica deste trabalho.

\section{REFERÊNCIAS}

BORGES, C.A.M. O conceito de desempenho de edificações e a sua importância para o setor da construção civil no Brasil. 2008. 263 f. Dissertação (Mestrado em Construção Civil) - Departamento de Engenharia de Construção Civil, Escola Politécnica da Universidade de São Paulo, São Paulo, 2008.

BRANDÃO, R.M.L. Levantamento das manifestações patológicas nas edificações com até cinco nos de idade, executadas no Estado de Goiás, 2007. 196 f. Dissertação (Mestrado em Engenharia) - Curso de Mestrado em Engenharia Civil, Escola de Engenharia Civil, Universidade Federal de Goiás, Goiânia, 2007.

FIESS, J.R.F.; OLIVEIRA, L.A.; RBIANCHI, A.C.; THOMAZ, E. Causas da ocorrência de manifestações patológicas em conjuntos habitacionais do estado de São Paulo. In: I CONFERÊNCIA LATINOAMERICANA DE CONSTRUÇÃO SUSTENTÁVEL, ENCONTRO NACIONAL DE TECNOLOGIA DO AMBIENTE CONSTRUÍDO, 10., 2004, São Paulo. Anais... São Paulo, 2004. p. 1-6.

HELENE, P. Manual de reparo, proteção e reforço de estruturas de concreto. São Paulo: Red Rehabilitar, 2003.

MELHADO, S.B. Qualidade do Projeto na Construção de Edifícios: Aplicação ao Caso das Empresas de Incorporação e Construção. São Paulo, EPUSP, 1994. (Tese Doutorado - EPUSP. Departamento de Engenharia de Construção Civil).

NÓBREGA JÚNIOR, C. L.; MELHADO, S. B. Coordenador de projetos de edificações: estudo e proposta para perfil, atividades e autonomia. São Paulo: EPUSP, 2013. 26 p. (Boletim Técnico da Escola Politécnica da USP, Departamento de Engenharia de Construção Civil, BT/PCC/579).

SILVA, M. V. M. F. P. da; NOVAES, C. C. A COORDENAÇÃO DE PROJETOS DE EDIFICAÇÕES: ESTUDOS DE CASO. Gestão \& Tecnologia de Projetos, [S.I.], v. 3, n. 1, p. 44-78, 2008. DOI: 10.4237/gtp.v3i1.28. Disponível em: http://www.revistas.usp.br/gestaodeprojetos/article/view/50927. Acesso em: 5 out. 2020. 
WOOD JR, T. Teoria Sistêmica Avançada e a Terceira Onda da Qualidade. Revista Politécnica da Usp, São Paulo, v. 211, p. 32-40, 1993. 